\title{
A Study of Some Tense Logics by Gentzen's Sequential Method
}

\author{
By \\ Hirokazu NisHimuRA*
}

\section{§1. Introduction}

Gentzen-style formulations of several fundamental modal logics like $T$, $S 4, S 5$, etc. are well-known now. See, e.g., Ohnishi and Matsumoto [6], Sato [9], Zeman [10], etc. Especially, Sato has established a close relationship between Gentzen-style formulations of modal calculi and Kripke-type semantics in a decisive way.

By the way, there is a strong analogy between classical tense logics and modal logics, which is also well-known. Indeed many techniques originally developed in modal calculi have been applied fruitfully to tense logics. For example, Gabbay [1] has used the so-called Lemmon-Scott or Makinson method to establish the completeness of many tense logics.

The main objective of the present paper is to present Gentzen-style formulations of some fundamental tense logics, say, $K_{t}$ and $K_{t} 4$, and then to prove the completeness of these logics with due regard to Gentzen-style formulations after the manner of Sato. Since the completeness of $K_{t}$ and $K_{t} 4$ is well-known, our main concern here rests in the relationship between our Gentzen-style systems and the ordinal semantics of tense logics.

Roughly speaking, traditional tense logics may be regarded as modal logics with two necessity-like operators, say, $G$ and $H$. However, we will see that the relationship of these two operators is much subtler than that of so-called bimodal logics.

\section{§2. Hilbert-type Systems}

Our formal language $L$ consists of the following symbols:

Communicated by S. Takasu, May 12, 1978.

* Research Institute for Mathematical Sciences, Kyoto University. 
(1) a countable set $P$ of propositional variables: $p, q, p^{\prime}, \ldots$

(2) classical connectives: $\neg, \supset$

(3) tense operators: $G, H$

(4) parentheses: (, )

The notion of a well-formed formula (or simply, a wff) is defined inductively as follows:

(1) Any propositional variable $p$ is a wff.

(2) If $\alpha$ and $\beta$ are wffs, so too are $(\neg \alpha),(\alpha \supset \beta),(G \alpha)$ and $(H \alpha)$.

In the rest of this paper our usage of parentheses is very loose, in so far as there is no danger of possible confusion.

For any wff $\alpha$, we define $\operatorname{Sub}(\alpha)$, the set of all subformulas of $\alpha$, inductively as follows:

(1) $\operatorname{Sub}(p)=\{p\}$

(2) $\operatorname{Sub}(\neg \alpha)=\operatorname{Sub}(\alpha) \cup\{\neg \alpha\}$

(3) $\operatorname{Sub}(\alpha \supset \beta)=\operatorname{Sub}(\alpha) \cup \operatorname{Sub}(\beta) \cup\{\alpha \supset \beta\}$

(4) $\operatorname{Sub}(G \alpha)=\operatorname{Sub}(\alpha) \cup\{G \alpha\}$

(5) $\operatorname{Sub}(H \alpha)=\operatorname{Sub}(\alpha) \cup\{H \alpha\}$

Now we review the traditional tense logics $K_{t}$ and $K_{t} 4$. We begin with the definition of $K_{t}$.

Axioms : (A1) $\alpha \supset(\beta \supset \alpha)$

(A2) $\quad(\alpha \supset(\beta \supset \gamma)) \supset((\alpha \supset \beta) \supset(\alpha \supset \gamma))$

(A3) $\quad(\neg \beta \supset \neg \alpha) \supset(\alpha \supset \beta)$

(G1) $\quad G(\alpha \supset \beta) \supset(G \alpha \supset G \beta)$

(H1) $\quad H(\alpha \supset \beta) \supset(H \alpha \supset H \beta)$

(G2) $\neg H \neg G \alpha \supset \alpha$

(H2) $\neg G \neg H \alpha \supset \alpha$

Rules : $\quad(\mathrm{MP}) \frac{\vdash \alpha \vdash \alpha \supset \beta}{\vdash \beta}$

(RG) $\frac{1-\alpha}{1-G \alpha}$

(RH) $\frac{\vdash \alpha}{\vdash \mathrm{H} \alpha}$

Now $K_{t} 4$ is defined to be the system obtained from $K_{t}$ by adding the following axioms. 


$$
\begin{array}{ll}
\text { (G3) } & G \alpha \supset G G \alpha \\
\text { (H3) } H \alpha \supset H H \alpha
\end{array}
$$

For more information on traditional tense logics in Hilbert style, see, e.g., Gabbay [1, 2], Prior [7] and Rescher and Urquhart [8].

\section{§3. Gentzen-type Systems I}

We now define Gentzen-type systems $G K_{t}$ and $G K_{t} 4$, which are equivalent to $K_{t}$ and $K_{t} 4$ respectively. We denote the set of all wffs by WFF. Following Sato [9], we define a sequent as an element in the set $2^{\mathrm{WFF}} \times 2^{\mathrm{WFF}}$. Namely, it is a pair of (possibly infinite) sets of wffs. In order to match with Gentzen's original notation, we will denote a sequent $\Gamma \rightarrow \Delta$ rather than $(\Gamma, \Delta)$. Some other notational conventions of Sato, which are almost self-explanatory, are adopted here. For example, $\Gamma \rightarrow \Delta, \Pi$ stands for $\Gamma \rightarrow \Delta \cup \Pi$.

We will also use the following notation:

(1) $\Gamma_{0} \rightarrow \Delta_{0} \subseteq \Gamma \rightarrow \Delta$ iff $\Gamma_{0} \subseteq \Gamma$ and $\Delta_{0} \subseteq \Delta$.

(2) $\Gamma_{0} \Subset \Gamma$ iff $\Gamma_{0} \subseteq \Gamma$ and $\Gamma_{0}$ is finite.

(3) $\Gamma_{0} \rightarrow \Delta_{0} \Subset \Gamma \rightarrow \Delta$ iff $\Gamma_{0} \Subset \Gamma$ and $\Delta_{0} \Subset \Delta$.

We now give the definition of the system $G K_{t}$.

Axioms : $\alpha \rightarrow \alpha$

$$
\begin{array}{ll}
\text { Rules : } & \frac{\Gamma \rightarrow \Delta}{\Pi, \Gamma \rightarrow \Delta, \Sigma} \text { (extension) } \\
& \Gamma \rightarrow \Delta, \alpha \quad \alpha, \Pi \rightarrow \Sigma \\
& \frac{\Gamma \rightarrow \Delta, \alpha}{\Gamma, \Pi \rightarrow \Delta, \Sigma}(\mathrm{cut}) \\
& \frac{}{\neg \alpha, \Gamma \rightarrow \Delta}(\neg \rightarrow) \\
& \frac{\alpha, \Gamma \rightarrow \Delta}{\Gamma \rightarrow \Delta, \neg \alpha}(\rightarrow \neg) \\
& \frac{\Gamma \rightarrow \Delta, \alpha \quad \beta, \Pi \rightarrow \Sigma}{\alpha \supset \beta, \Gamma, \Pi \rightarrow \Delta, \Sigma}(\supset \rightarrow) \\
& \frac{\alpha, \Gamma \rightarrow \Delta, \beta}{\Gamma \rightarrow \Delta, \alpha \supset \beta}(\rightarrow \supset) \\
& \frac{\Gamma \rightarrow \alpha, H \Delta}{G \Gamma \rightarrow G \alpha, \Delta}(\rightarrow G) \\
& \frac{\Gamma \rightarrow \alpha, G \Delta}{H \Gamma \rightarrow H \alpha, \Delta}(\rightarrow H)
\end{array}
$$


In the above rules $G \Gamma=\{G \alpha \mid \alpha \in \Gamma\}$ and $H \Gamma=\{H \alpha \mid \alpha \in \Gamma\}$ for any $\Gamma \subseteq \mathrm{WFF}$.

Now $G K_{t} 4$ is obtained from $G K_{t}$ by replacing the rules $(\rightarrow G)$ and $(\rightarrow H)$ by the following rules respectively.

$$
\begin{gathered}
\frac{G \Gamma, \Gamma \rightarrow \alpha, H \Delta, H \Sigma}{G \Gamma \rightarrow G \alpha, \Delta, H \Sigma}(\rightarrow G)_{4} \\
\frac{H \Gamma, \Gamma \rightarrow \alpha, G \Delta, G \Sigma}{H \Gamma \rightarrow H \alpha, \Delta, G \Sigma}(\rightarrow H)_{4}
\end{gathered}
$$

It is important to notice that $(\rightarrow G)$ and $(\rightarrow H)$ are admissible rules in $G K_{t} 4$.

We call a sequent $\Gamma \rightarrow \Delta$ finite if both $\Gamma$ and $\Delta$ are finite. Then it is easy to prove the following lemma.

Lemma 3.1. If a finite sequent $\Gamma \rightarrow \Delta$ is provable in $G K_{t}$ (in $G K_{t} 4$, resp.), then each sequent occurring in any proof of $\Gamma \rightarrow \Delta$ is finite.

Theorem 3.2. If $\vdash \Gamma \rightarrow \Delta$ in $G K_{t}$ (in $G K_{t} 4$, resp.), then there exist some $\Gamma_{0} \rightarrow \Delta_{0} \Subset \Gamma \rightarrow \Delta$ such that $\vdash \Gamma_{0} \rightarrow \Delta_{0}$ in $G K_{t}$ (in $G K_{t} 4$, resp.).

Proof. By induction on the number $n$ of sequents occurring in the proof of $\Gamma \rightarrow \Delta$.

It is easy to see the equivalence of $K_{t}$ and $G K_{t}\left(K_{t} 4\right.$ and $G K_{t} 4$, resp.).

Theorem 3.3. For any wff $\alpha, \vdash \alpha$ in $K_{t}$ (in $K_{t} 4$, resp.) iff $\vdash \rightarrow \alpha$ in $G K_{t}$ (in $G K_{t} 4$, resp.).

Corollary 3.4. Let $\Gamma \subseteq \mathrm{WFF}$ and $\alpha \in \mathrm{WFF}$. Then $\Gamma \vdash \alpha$ in $K_{t}$ (in $K_{t} 4$, resp.) iff $\vdash \Gamma \rightarrow \alpha$ in $G K_{t}$ (in $G K_{t} 4$, resp.).

The following example shows that our sequential systems $G K_{t}$ and $G K_{t} 4$ are not cut-free.

$$
\frac{(\neg \rightarrow) \frac{p \rightarrow p}{(\rightarrow \neg) \frac{\neg p, p \rightarrow}{p \rightarrow \neg \neg p}} \frac{\frac{H \neg p \rightarrow H \neg p}{\rightarrow \neg H \neg p, H \neg p}}{\frac{\rightarrow \neg G \neg H \neg p, \neg p}{\neg \neg p \rightarrow G \neg H \neg p}(\rightarrow G)}(\neg \rightarrow)}{\text { (cut) }}
$$

Thus we conclude this section by the following theorem.

Theorem 3.5. The cut-elimination theorem does fail for $G K_{t}$ and $G K_{t} 4$.

\section{§4. Completeness}

First of all, we review the semantics for tense logic. By a T-structure, we 
mean a triple $(S, R, D)$, where

(1) $S$ is a set (called the "time").

(2) $R$ is a binary relation on $S$ (the earlier-later relation).

(3) $D$ is a function from $P \times S$ to $\{0,1\}$. That is, $D$ assigns a truth-value to each propositional variable at each moment (an element of $S$ is called a moment).

Given a T-structure $(S, R, D)$, the truth-value $V(\alpha: t)$ of a wff $\alpha$ at a moment $t$ is defined inductively as follows:

(V1) $V(p: t)=D(p, t)$ for any propositional variable $p$.

(V2) $V(\neg \alpha: t)=1$ iff $V(\alpha: t)=0$.

(V3) $V(\alpha \supset \beta: t)=1$ iff $V(\alpha: t)=0$ or $V(\beta: t)=1$.

(V4) $V(G \alpha: t)=1$ iff for any $s \in S$ such that $t R s, V(\alpha: s)=1$.

(V5) $V(H \alpha: t)=1$ iff for any $s \in S$ such that $s R t, V(\alpha: s)=1$.

We also define $V(\Gamma \rightarrow \Delta: t)$, where $\Gamma \rightarrow \Delta$ is a sequent, as follows:

(V6) $V(\Gamma \rightarrow \Delta: t)=1$ iff $V(\alpha: t)=1$ for any $\alpha \in \Gamma$ and $V(\beta: t)=0$ for any $\beta \in \Delta$.

By a T-model, we mean a 4-tuple $(S, R, D, o)$, where

(1) $(S, R, D)$ is a T-structure.

(2) $o$ is an element of $S$ (called the "present moment").

A T-structure $(S, R, D)$ (a T-model $(S, R, D, o)$, resp.) is called a T4-structure (T4-model, resp.) if $R$ is a transitive relation.

We say that:

(1) A T-model $(S, R, D, o)$ realizes a sequent $\Gamma \rightarrow \Delta$ if $V(\Gamma \rightarrow \Delta: o)=1$.

(2) A sequent $\Gamma \rightarrow \Delta$ is T-realizable (T4-realizable, resp.) if $\Gamma \rightarrow \Delta$ can be realized by some T-model (T4-model, resp.).

(3) A sequent $\Gamma \rightarrow \Delta$ is T-valid (T4-valid, resp.) if it is not T-realizable (T4realizable, resp.).

We say that:

(1) A sequent $\Gamma \rightarrow \Delta$ is G-provable (G4-provable, resp.) if it is provable in $G K_{t}$ (in $G K_{t} 4$, resp.).

(2) A sequent $\Gamma \rightarrow \Delta$ is G-consistent (G4-consistent, resp.) if it is not G-provable (G4-provable, resp.).

With these definitional preparations, we can present the following theorem. 
Theorem 4.1 (Soundness Theorem). Any G-provable sequent (G4provable sequent, resp.) is T-valid (T4-valid, resp.).

Corollary 4.2. If $\vdash \alpha$ in $K_{t}$ (in $K_{t} 4$, resp.), then $V(\alpha: o)=1$ for any T-model (T4-model, resp.) $(S, R, D, o)$.

Proof. This is immediate from Theorem 3.3 and Theorem 4.1.

Corollary 4.3 (Consistency of $G K_{t}$ and $G K_{t} 4$ ). The empty sequent $\rightarrow$ is not provable in $G K_{t}$ (in $G K_{t} 4$, resp.).

We now deal with completeness theorems. It is easy to see that the following lemma holds.

Lemma 4.4 (Lindenbaum's Lemma). Let it be that $\wedge \Gamma \rightarrow \Delta$ in $G K_{t}$ (in $G K_{t} 4$, resp.) and $\Omega$ is a set of wffs such that $\Gamma \cup \Delta \subseteq \Omega$. Then there exist $\tilde{\Gamma}$, $\tilde{\Delta}$ such that:

(1) $\uparrow \tilde{\Gamma} \rightarrow \tilde{\Delta}$ in $G K_{t}$ (in $G K_{t} 4$, resp.).

(2) $\tilde{\Gamma} \rightarrow \tilde{\Delta} \supseteq \Gamma \rightarrow \Delta$.

(3) $\tilde{\Gamma} \cup \tilde{\Delta}=\Omega$.

A set $\Omega$ of wffs is said to be closed under subformulas if $\operatorname{Sub}(\alpha) \subseteq \Omega$ for any $\alpha \in \Omega$. Now take any such $\Omega$ and fix it. A sequent $\Gamma \rightarrow \Delta$ is said to be $\Omega, \mathrm{G}-$ compleie $(\Omega, \mathrm{G} 4$-complete, resp.) if $\Gamma \rightarrow \Delta$ is G-consistent (G4-consistent, resp.) and $\Gamma \cup \Delta=\Omega$. We define $C(\Omega)$ and $C_{4}(\Omega)$ as follows:

(1) $C(\Omega)=\{\Gamma \rightarrow \Delta \mid \Gamma \rightarrow \Delta$ is $\Omega$, G-complete $\}$.

(2) $C_{4}(\Omega)=\{\Gamma \rightarrow \Delta \mid \Gamma \rightarrow \Delta$ is $\Omega$, G4-complete $\}$.

It is easy to see that for any $\Gamma \rightarrow \Delta \in C(\Omega), \Gamma \cap \Delta=\emptyset$ because $\Gamma \rightarrow \Delta$ is Gconsistent. Similarly for any $\Gamma \rightarrow \Delta \in C_{4}(\Omega), \Gamma \cap \Delta=\emptyset$. For any $\Gamma \subseteq \mathrm{WFF}$, we denote by $\Gamma_{G}$ and $\Gamma_{H}$ the sets $\{\alpha \mid G \alpha \in \Gamma\}$ and $\{\alpha \mid H \alpha \in \Gamma\}$ respectively. We now define the universal T-structure $U(\Omega)=(S, R, D)$ as follows:

(1) $S=C(\Omega)$.

(2) $(\Gamma \rightarrow \Delta) R\left(\Gamma^{\prime} \rightarrow \Delta^{\prime}\right)$ iff $\Gamma_{G} \subseteq \Gamma^{\prime}$ and $\Gamma_{H}^{\prime} \subseteq \Gamma$.

(3) $D(p, \Gamma \rightarrow \Delta)=1$ iff $p \in \Gamma$.

Similarly we define the universal T4-structure $U_{4}(\Omega)=\left(S^{\prime}, R^{\prime}, D^{\prime}\right)$ as follows:

(1) $S^{\prime}=C_{4}(\Omega)$.

(2) $(\Gamma \rightarrow \Delta) R^{\prime}\left(\Gamma^{\prime} \rightarrow \Delta^{\prime}\right)$ iff $\Gamma_{G} \subseteq \Gamma^{\prime}, \Gamma_{G} \subseteq \Gamma_{G}^{\prime}, \Gamma_{H}^{\prime} \subseteq \Gamma$ and $\Gamma_{H}^{\prime} \subseteq \Gamma_{H}$.

(3) $D^{\prime}(p, \Gamma \rightarrow \Delta)=1$ iff $p \in \Gamma$. 
It is easy to see that $U(\Omega)\left(U_{4}(\Omega)\right.$, resp.) is indeed a T-structure (T4-structure, resp.).

Theorem 4.5 (Fundamental Theorem of Universal Structure). For any $\alpha \in \Omega$ and $\Gamma \rightarrow \Delta \in U(\Omega)\left(U_{4}(\Omega)\right.$, resp. $), V(\alpha: \Gamma \rightarrow \Delta)=1$ if $\alpha \in \Gamma$ and $V(\alpha: \Gamma \rightarrow \Delta)$ $=0$ if $\alpha \in \Delta$.

Proof. By induction on the construction of wffs.

(a) $\alpha$ is a propositional variable: Immediate from the definition of $D\left(D^{\prime}\right.$, resp.).

(b) $\alpha=\neg \beta$ : Suppose $\alpha \in \Gamma$. It is sufficient to show that $\propto \Gamma \rightarrow \Delta, \beta$, which implies $\beta \in \Delta$ because of the maximality of $\Gamma \rightarrow \Delta$. In this case we can conclude that $V(\alpha: \Gamma \rightarrow \Delta)=1$ by induction hypothesis. Suppose, for the sake of contradiction, that $\vdash \Gamma \rightarrow \Delta, \beta$. Then we can show that $\vdash \Gamma \rightarrow \Delta$ as follows:

$$
\frac{\Gamma \rightarrow \Delta, \beta}{\neg \beta, \Gamma \rightarrow \Delta}(\neg \rightarrow)
$$

This is a contradiction. The case $\alpha \in \Delta$ can be treated in a similar manner.

(c) $\alpha=\beta \supset \gamma$ : Suppose $\alpha \in \Gamma$. It is sufficient to show that $\alpha \Gamma \rightarrow \Delta, \beta$ or $\uparrow \gamma, \Gamma \rightarrow \Delta$, which implies that $\beta \in \Delta$ or $\gamma \in \Gamma$. In any case $V(\alpha: \Gamma \rightarrow \Delta)=1$ by induction hypothesis. Suppose, for the sake of contradiction, that $\vdash \Gamma \rightarrow \Delta, \beta$ and $\vdash \gamma, \Gamma \rightarrow \Delta$. Then we can show that $\vdash \Gamma \rightarrow \Delta$ as follows:

$$
\frac{\Gamma \rightarrow \Delta, \beta \quad \gamma, \Gamma \rightarrow \Delta}{\beta \supset \gamma, \Gamma \rightarrow \Delta}(\supset \rightarrow)
$$

This is a contradiction. Suppose $\alpha \in \Delta$. It is sufficient to show that $\chi \beta, \Gamma \rightarrow \Delta$, $\gamma$, which implies $\beta \in \Gamma$ and $\gamma \in \Delta$, because of the maximality of $\Gamma \rightarrow \Delta$. So we can conclude $V(\alpha: \Gamma \rightarrow \Delta)=0$ by induction hypothesis. Suppose, for the sake of contradiction, that $1-\beta, \Gamma \rightarrow \Delta, \gamma$. Then we can show that $\vdash \Gamma \rightarrow \Delta$ as follows:

$$
\frac{\beta, \Gamma \rightarrow \Delta, \gamma}{\Gamma \rightarrow \Delta, \beta \supset \gamma}(\rightarrow \supset)
$$

This is a contradiction.

(d) $\alpha=G \beta$ : Suppose $\alpha \in \Gamma$. That $V(\alpha: \Gamma \rightarrow \Delta)=1$ follows directly from the definition of $R$ or $R^{\prime}$.

Now suppose that $\alpha \in \Delta$.

For $U(\Omega)$ : We show that the sequent $\Gamma_{G} \rightarrow \beta,\{H \gamma \mid H \gamma \in \Omega$ and $\gamma \in \Delta\}$ is Gconsistent. We assume, for the sake of contradiction, that $\vdash \Gamma_{G} \rightarrow \beta,\{H \gamma \mid$ $H \gamma \in \Omega$ and $\gamma \in \Delta\}$. Then we can show that $\vdash \Gamma \rightarrow \Delta$ as follows: 


$$
\frac{\frac{\Gamma_{G} \rightarrow \beta,\{H \gamma \mid H \gamma \in \Omega \text { and } \gamma \in \Delta\}}{G \Gamma_{G} \rightarrow G \beta,\{\gamma \mid H \gamma \in \Omega \text { and } \gamma \in \Delta\}}}{\Gamma \rightarrow \Delta}(\rightarrow G)
$$

This is a contradiction. So we can conclude that the sequent $\Gamma_{G} \rightarrow \beta,\{H \gamma \mid$ $H \gamma \in \Omega$ and $\gamma \in \Delta\}$ is G-consistent. By Lemma 6.4 this sequent can be extended to some $\Omega$, G-complete sequent $\Gamma^{\prime} \rightarrow \Delta^{\prime}$. It is easy to see that $(\Gamma \rightarrow \Delta) R\left(\Gamma^{\prime} \rightarrow \Delta^{\prime}\right)$ and $V\left(\beta: \Gamma^{\prime} \rightarrow \Delta^{\prime}\right)=0$. Therefore $V(\alpha: \Gamma \rightarrow \Delta)=0$.

For $U_{4}(\Omega)$ : We show that the sequent $\Gamma_{G}, G \Gamma_{G} \rightarrow \beta,\{H \gamma \mid H \gamma \in \Omega$ and $\gamma \in \Delta\}$, $H \Delta_{H}$ is G4-consistent. We assume, for the sake of contradiction, that this sequent is G4-provable. Then we see that $\Gamma \rightarrow \Delta$ is also G4-provable as the following proof-figure shows:

$$
\frac{\frac{G \Gamma_{G}, \Gamma_{G} \rightarrow \beta,\{H \gamma \mid H \gamma \in \Omega \text { and } \gamma \in \Delta\}, H \Delta_{H}}{G \Gamma_{G} \rightarrow G \beta,\{\gamma \mid H \gamma \in \Omega \text { and } \gamma \in \Delta\}, H \Delta_{H}}}{\Gamma \rightarrow \Delta}(\rightarrow G)_{4}
$$

This is a contradiction. So we can conclude that the sequent $G \Gamma_{G}, \Gamma_{G} \rightarrow \beta$, $\{H \gamma \mid H \gamma \in \Omega$ and $\gamma \in \Delta\}, H \Delta_{H}$ is G4-consistent. By Lemma 6.4 this sequent can be extended to some $\Omega$, G4-complete sequent $\Gamma^{\prime} \rightarrow \Delta^{\prime}$. It is easy to see that $(\Gamma \rightarrow \Delta) R^{\prime}\left(\Gamma^{\prime} \rightarrow \Delta^{\prime}\right)$ and $V\left(\beta: \Gamma^{\prime} \rightarrow \Delta^{\prime}\right)=0$. Therefore $V(\alpha: \Gamma \rightarrow \Delta)=0$.

(e) $\alpha=H \beta$ : Similar to the case (d).

Several results follow directly from this theorem.

Theorem 4.6 (Generalized Completeness Theorem). Any G-consistent (G4-consistent, resp.) sequent is T-realizable (T4-realizable, resp.).

Proof. Immediate from Lemma 4.4 and Theorem 4.5.

Theorem 4.7 (Compactness Theorem). For any sequent $\Gamma \rightarrow \Delta, \Gamma \rightarrow \Delta$ is T-realizable (T4-realizable, resp.) iff for any $\Gamma_{0} \rightarrow \Delta_{0} \Subset \Gamma \rightarrow \Delta$ is T-realizable (T4-realizable, resp.).

Theorem 4.8 (Completeness and Decidability Theorem). For any finite sequent $\Gamma \rightarrow \Delta, \Gamma \rightarrow \Delta$ is G-provable (G4-provable, resp.) iff $\Gamma \rightarrow \Delta$ holds in all $\mathrm{T}$-models (T4-models, resp.) whose cardinality $\leq 2^{n}$, where $n$ is the cardinality of $\cup_{\alpha \in \Gamma \cup \Delta} \operatorname{Sub}(\alpha)$.

\section{§5. Gentzen-type Systems II}

In Section 3 we have introduced Gentzen-type systems $G K_{t}$ and $G K_{t} 4$, which 
was shown to be deductively equivalent to traditional tense logics $K_{t}$ and $K_{t} 4$ in Hilbert style respectively in Section 4. However, strictly speaking, $G K_{t}$ and $G K_{t} 4$ are somewhat crude since in the rules $(\rightarrow G),(\rightarrow H),(\rightarrow G)_{4}$ and $(\rightarrow H)_{4}$ some subformulas of the upper sequent may disappear in the lower sequent. That is, $G K_{t}$ and $G K_{t} 4$ can not necessarily enjoy the usual property of ordinal Gentzen-type systems that the totality of subformulas of a sequent increase as we proceed downward in a proof-figure without a cut. But this defect of $G K_{t}$ and $G K_{t} 4$ is rather superficial than crucial, and the main purpose of this section is to introduce more elaborated Gentzen-type systems $G H K_{t}$ and $G H K_{t} 4$, which are to be shown to be deductively equivalent to $G K_{t}$ and $G K_{t} 4$ (and so to $K_{t}$ and $K_{t} 4$ ) respectively.

We now define $G H K_{t}$. In $G H K_{t}$, a sequent is defined to be an element of the set $2^{\mathrm{WFF}} \times 2^{\mathrm{WFF}} \times 2^{\mathrm{WFF}} \times 2^{\mathrm{WFF}} \times 2^{\mathrm{WFF}} \times 2^{\mathrm{WFF}}$. Thus a sequent is of the form $\left(\Pi_{1}, \Gamma, \Pi_{2}, \Sigma_{1}, \Delta, \Sigma_{2}\right)$. However, we denote this as $\Pi_{1} ; \Gamma ; \Pi_{2} \rightarrow \Sigma_{1}$; $\Delta ; \Sigma_{2}$. Moreover we denote $; \Gamma ; \rightarrow ; \Delta ; \quad(=(\varnothing, \Gamma, \emptyset, \emptyset, \Delta, \emptyset))$ simply as $\Gamma \rightarrow \Delta$. A sequent of this form will be called proper. Other sequents will be called improper.

We define $G H K_{t}$ as follows:

Axioms : $\alpha \rightarrow \alpha$

$$
\begin{aligned}
& \alpha ; ; \rightarrow \alpha ; \\
& ; ; \alpha \rightarrow ; \alpha
\end{aligned}
$$

Rules : $\frac{\Pi_{1} ; \Gamma ; \Pi_{2} \rightarrow \Sigma_{1} ; \Delta ; \Sigma_{2}}{\Pi_{1}^{\prime}, \Pi_{1} ; \Gamma^{\prime}, \Gamma ; \Pi_{2}^{\prime}, \Pi_{2} \rightarrow \Sigma_{1}^{\prime}, \Sigma_{1} ; \Delta^{\prime}, \Delta ; \Sigma_{2}^{\prime}, \Sigma_{2}}$ (extension)

$$
\begin{aligned}
& \frac{\Pi_{1} ; \Gamma ; \Pi_{2} \rightarrow \Sigma_{1} ; \Delta, \alpha ; \Sigma_{2} \Pi_{1}^{\prime} ; \alpha, \Gamma^{\prime} ; \Pi_{2}^{\prime} \rightarrow \Sigma_{1}^{\prime} ; \Delta^{\prime} ; \Sigma_{2}^{\prime}}{\Pi_{1}, \Pi_{1}^{\prime} ; \Gamma, \Gamma^{\prime} ; \Pi_{2}, \Pi_{2}^{\prime} \rightarrow \Sigma_{1}, \Sigma_{1}^{\prime} ; \Delta, \Delta^{\prime} ; \Sigma_{2}, \Sigma_{2}^{\prime}} \text { (cut) } \\
& \frac{\Pi_{1} ; \Gamma ; \alpha, \Pi_{2} \rightarrow \Sigma_{1} ; \Delta ; \Sigma_{2}}{\Pi_{1} ; \Gamma, G \alpha ; \Pi_{2} \rightarrow \Sigma_{1} ; \Delta ; \Sigma_{2}}(G \rightarrow: \text { out }) \\
& \frac{\Pi_{1} ; \Gamma ; \Pi_{2} \rightarrow \Sigma_{1} ; \Delta ; \alpha, \Sigma_{2}}{\Pi_{1} ; \Gamma ; \Pi_{2} \rightarrow \Sigma_{1} ; \Delta, G \alpha ; \Sigma_{2}}(\rightarrow G: \text { out }) \\
& \frac{\Pi_{1}, \alpha ; \Gamma ; \Pi_{2} \rightarrow \Sigma_{1} ; \Delta ; \Sigma_{2}}{\Pi_{1} ; H \alpha, \Gamma ; \Pi_{2} \rightarrow \Sigma_{1} ; \Delta ; \Sigma_{2}}(H \rightarrow: \text { out }) \\
& \frac{\Pi_{1} ; \Gamma ; \Pi_{2} \rightarrow \Sigma_{1}, \alpha ; \Delta ; \Sigma_{2}}{\Pi_{1} ; \Gamma ; \Pi_{2} \rightarrow \Sigma_{1} ; H \alpha, \Delta ; \Sigma_{2}}(\rightarrow H: \text { out }) \\
& \frac{\Pi_{1} ; \Gamma ; \Pi_{2} \rightarrow \Sigma_{1} ; \Delta, \alpha ; \Sigma_{2}}{\Pi_{1} ; \neg \alpha, \Gamma ; \Pi_{2} \rightarrow \Sigma_{1} ; \Delta ; \Sigma_{2}}(\neg \rightarrow) \\
& \frac{\Pi_{1} ; \alpha, \Gamma ; \Pi_{2} \rightarrow \Sigma_{1} ; \Delta ; \Sigma_{2}}{\Pi_{1} ; \Gamma ; \Pi_{2} \rightarrow \Sigma_{1} ; \Delta, 7 \alpha ; \Sigma_{2}}(\rightarrow \neg)
\end{aligned}
$$




$$
\begin{aligned}
& \frac{\Pi_{1} ; \Gamma ; \Pi_{2} \rightarrow \Sigma_{1} ; \Delta, \alpha ; \Sigma_{2} \quad \Pi_{1}^{\prime} ; \beta, \Gamma^{\prime} ; \Pi_{2}^{\prime} \rightarrow \Sigma_{1}^{\prime} ; \Delta^{\prime} ; \Sigma_{2}^{\prime}}{\Pi_{1}, \Pi_{1}^{\prime} ; \alpha \supset \beta, \Gamma, \Gamma^{\prime} ; \Pi_{2}, \Pi_{2}^{\prime} \rightarrow \Sigma_{1}, \Sigma_{1}^{\prime} ; \Delta, \Delta^{\prime} ; \Sigma_{2}, \Sigma_{2}^{\prime}}(\supset \rightarrow) \\
& \frac{\Pi_{1} ; \alpha, \Gamma ; \Pi_{2} \rightarrow \Sigma_{1} ; \Delta, \beta ; \Sigma_{2}}{\Pi_{1} ; \Gamma ; \Pi_{2} \rightarrow \Sigma_{1} ; \Delta, \alpha \supset \beta ; \Sigma_{2}}(\rightarrow \supset) \\
& \frac{; \Gamma ; \rightarrow \Delta ; \alpha ;}{; ; \Gamma \rightarrow ; \Delta ; \alpha} \text { (r-trans) } \\
& \frac{; \Gamma ; \rightarrow ; \alpha ; \Delta}{\Gamma ; ; \rightarrow \alpha ; \Delta ;} \text { (1-trans) }
\end{aligned}
$$

Now, $G H K_{t} 4$ is obtained from $G H K_{t}$ by replacing the rules (r-trans) and (l-trans) by the following rules respectively.

$$
\begin{aligned}
& \frac{; \Gamma ; \Gamma \rightarrow \Delta, \Sigma ; \alpha ;}{; ; \Gamma \rightarrow \Sigma ; \Delta ; \alpha}(\text { r-trans })_{4} \\
& \frac{\Gamma ; \Gamma ; \rightarrow ; \alpha ; \Delta, \Sigma}{\Gamma ; \text { (1-trans })_{4}}
\end{aligned}
$$

Now we should prove the equivalence of $G K_{t}$ and $G H K_{t}\left(G K_{t} 4\right.$ and $G H K_{t} 4$, resp.).

Theorem 5.1. Let $\Gamma \rightarrow \Delta$ be a proper sequent. Then $\vdash \Gamma \rightarrow \Delta$ in $G K_{t}$ (in $G K_{t} 4$, resp.) iff $\vdash \Gamma \rightarrow \Delta$ in $G H K_{t}$ (in $G H K_{t} 4$, resp.).

Proof. We deal only with the equivalence of $G K_{t} 4$ and $G H K_{t} 4$.

If part: By induction on the construction of the proof, we prove that if $\vdash \Pi_{1} ; \Gamma ; \Pi_{2} \rightarrow \Sigma_{1} ; \Delta ; \Sigma_{2}$ in $G H K_{t} 4$, then $\vdash H \Pi_{1}, \Gamma, G \Pi_{2} \rightarrow H \Sigma_{1}, \Delta, G \Sigma_{2}$ in $G K_{t} 4$. The proof is almost straight, and so is left to the reader. But we comment that (r-trans) $)_{4}$ and (l-trans) $)_{4}$ correspond to $(\rightarrow G)_{4}$ and $(\rightarrow H)_{4}$ respectively. Only if part: For any $\Gamma \subseteq \mathrm{WFF}$, we denote the sets $\{\alpha \mid G \alpha \in \Gamma\}$ and $\{\alpha \mid H \alpha \in \Gamma\}$ by $\Gamma_{G}$ and $\Gamma_{H}$ respectively. We denote $\Gamma-\Gamma_{G} \cup \Gamma_{H}$ by $\Gamma_{J}$. By induction on the construction of the proof, we prove that $\vdash \Gamma \rightarrow \Delta$ in $G K_{t} 4$, then $\vdash \Gamma_{H} ; \Gamma_{J} ; \Gamma_{G}$ $\rightarrow \Delta_{H} ; \Delta_{J} ; \Delta_{G}$ in $G H K_{t} 4$. Since the proof is almost direct, it is left to the reader.

As corollaries of this theorem,

Corollary 5.2. $\vdash \Pi_{1} ; \Gamma ; \Pi_{2} \rightarrow \Sigma_{1} ; \Delta ; \Sigma_{2}$ in $G H K_{t}$ (in $G H K_{t} 4$, resp.) iff $\vdash H \Pi_{1}, \Gamma, G \Pi_{2} \rightarrow H \Sigma_{1}, \Delta, G \Sigma_{2}$ in $G K_{t}$ (in $G K_{t} 4$, resp.).

Corollary 5.3. A sequent $\Pi_{1} ; \Gamma ; \Pi_{2} \rightarrow \Sigma_{1} ; \Delta ; \Sigma_{2}$ is provable without a cut in $G H K_{t}$ (in $G H K_{t} 4$, resp.) iff the sequent $H \Pi_{1}, \Gamma, G \Pi_{2} \rightarrow H \Sigma_{1}, \Delta, G \Sigma_{2}$ is provable without a cut in $G K_{t}$ (in $G K_{t} 4$, resp.).

Corollary 5.4. The cut-elimination theorem does fail for $G H K_{t}$ and $G \mathrm{~K}_{t} 4$. 
Proof. Immediate from Corollary 5.3 and Theorem 3.5.

\section{Acknowledgements}

I would like to thank professors Satoru Takasu, Masahiko Sato and Takeshi Hayashi.

\section{References}

[1] Gabbay, D. M., Model theory for tense logics, Annals of Math. Logic, 8 (1975), 185-236.

[2] - Investigations in modal and tense logics with applications to problems in philosophy and linguistics, D. Reidel, 1976.

[ 3 ] Gentzen, G., Untersuchungen über das logische Schliessen I, II, Math. Z., 39 (1935), 176-210, 405-431.

[4] Hughes, G. E. and Cresswell, M. J., An introduction to modal logic, Methuen, 1968.

[5] Matsumoto, K., Sûri Ronrigaku (Mathematical logic), (in Japanese), Kyoritsu, 1965.

[6] Ohnishi, M. and Matsumoto, K., Gentzen method in modal calculi, Osaka Math. J., 9 (1957), 113-130, 11 (1959), 115-120.

[7] Prior, A. N., Past, present and future, Oxford, 1967.

[8] Rescher, N. and Urquhart, A., Temporal logic, Springer, 1971.

[9] Sato, M., A study of Kripke-type models for some modal logics by Gentzen's sequential method, Publ. RIMS, Kyoto Univ., 13 (1977), 381-468.

[10] Zeman, J. J., Modal logic, Oxford, 1973. 
